\title{
Manifestaciones orales de la incontinencia pigmentaria (Síndrome de BlochSulzberger). Reporte de caso
}

\author{
Oral manifestations of pigmentary incontinence \\ (BlochSulzberger syndrome). Case report \\ Yuri Castro-Rodríguez' ORCID: 0000-0002-9587-520X \\ Jose Cósar-Quiroz² ORCID: 0000-0001-5972-4095 \\ Elizabhet Julcamoro-Grados² ORCID: 0000-0002-8704-8167
}

\section{Resumen}

Paciente de sexo femenino de 13 años de edad que acudió al servicio de Odontopediatría del Instituto de Salud Oral del Hospital Central de la Fuerza Aérea de Perú en el mes de setiembre del año 2018 debido a una renovación en la prótesis parcial provisional que utilizaba desde hace cinco meses. Al examen clínico extraoral se evidenció lesiones cicatrizales en la piel de la región nasal; durante la evaluación intraoral se evidenció la pérdida de múltiples dientes, rebordes edéntulos amplios y delgados, alteraciones en la morfología dentaria, así como discordancia de la erupción dentaria con la edad de la paciente. Al examen radiográfico se evidenció alteración y retardo en la erupción dentaria, impactación de un diente en la mandíbula, falta de desarrollo radicular y persistencia de dientes temporales. El diagnóstico médico definitivo fue el Síndrome de Bloch - Sulzberger también denominado como "incontinencia pigmentaria". Este síndrome se caracteriza por presentar una variedad de manifestaciones orales como: agenesia dentaria, alteración en la morfología dentaria, dientes retenidos y/o impactados así como alteración y retraso en la erupción dentaria. A nivel sistémico este síndrome presenta lesiones que afectan a la piel, pelo, visión, sistema nervioso central, entre otros.

Palabras clave: síndrome, erupción dental, agenesia dental, diente impactado.

Facultad de Ciencias de la Salud. Universidad Privada Juan Pablo II. Perú.

Facultad de Odontología. Sociedad Científica de Estudiantes de Odontología. Universidad Nacional Mayor de San Marcos. Perú.

Fecha de recibido: 28.02.2019 - Fecha de aceptado: 27.07.2019 


\section{Abstract}

A 13-year-old female patient went to the pediatric dentistry service of the Oral Health Institute of the Central Air Force Hospital of Peru in September of 2018. She sought care to have her temporary partial prosthesis renewed. The extraoral clinical examination showed scar lesions on the skin of the nasal region; the intraoral evaluation showed the loss of multiple teeth, wide and thin edentulous ridges, alterations in dental morphology, as well as the inconsistency of the dental eruption with the patient's age. The radiographic examination showed alteration and delay in dental eruption, impaction of a tooth in the jaw, lack of root development and persistence of temporary teeth. The definitive medical diagnosis was BlochSulzberger Syndrome, also referred to as "pigmentary incontinence". This syndrome is characterized by presenting a variety of oral manifestations such as dental agenesis, alteration in dental morphology, retained and/or impacted teeth as well as alteration and delay in dental eruption. At a systemic level, this syndrome has lesions that affect the skin, hair, vision, central nervous system, among others.

Keywords: syndrome, dental eruption, dental agenesis, tooth, impacted.

\section{Introducción}

El Síndrome de Bloch-Sulzberger o incontinencia pigmentaria (IP) es considerada como una "genodermatosis" asociada al cromosoma X ${ }^{(1)}$. Se caracteriza por la presencia de lesiones ampollares distribuidas en la piel de forma generalizada. Clínicamente presenta cuatro estadios: vesiculo-ampollar, verrugoso, hiperpigmentado

\section{Resumo}

Paciente do sexo feminino, 13 anos, que compareceu ao Serviço de Odontopediatria do Instituto de Saúde Bucal do Hospital Central da Força Aérea do Peru, em setembro de 2018, devido à renovação da prótese parcial provisória utilizada por ela. por cinco meses. O exame clínico extraoral mostrou lesôes cicatriciais na pele da região nasal; Durante a avaliaçáo intraoral, a perda de múltiplos dentes, cristas edêntulas, extensas e finas, alterações na morfologia dentária, bem como a incongruência da erupção dentária com a idade do paciente foi evidente. O exame radiográfico mostrou alteração e atraso na erupção dentária, impactação de um dente na mandíbula, falta de desenvolvimento radicular e persistência de dentes temporários. O diagnóstico médico definitivo foi a síndrome de Bloch - Sulzberger, também conhecida como "incontinência pigmentar". A síndrome é caracterizada por apresentar uma variedade de manifestaçóes bucais, como: agenesia dentária, alteração na morfologia dentária, retenção e / ou impacto dos dentes, bem como alteração e atraso na erupção dentária. Em nível sistêmico, essa síndrome apresenta lesóes que afetam a pele, o cabelo, a visão, o sistema nervoso central, entre outros.

Palavras-chave: síndrome, erupção cutânea, agenesia dentária, dente impactado.

y atrófico. Los pacientes raras veces desarrollan las cuatro formas de manera progresiva y en algunas ocasiones estos se superponen.

El síndrome afecta diversos órganos y se caracteriza por la formación de ampollas en forma de bandas, distribuirse de forma lineal o estar diversificadas y que comúnmente se encuentran presentes poco después del nacimiento ${ }^{(2)}$. Las lesiones afectan a la piel, 
pelo, dientes, ojos, sistema nervioso central, entre otros ${ }^{(3)}$.

\section{Antecedentes}

Inicialmente fue descrita por Garrod como "una variedad de idiocia mongólica con una pigmentación peculiar" (4,5). Posteriormente fue definida por Bardach (1925), Bloch (1926), Sulzberger (1928) y Siemens (1929); por este motivo también es denominada como Síndrome de Bloch - Siemens ${ }^{(6,7)}$.

Es más común que las mujeres se vean afectadas (95\%) debido a que en los varones se ve acompañada con una elevada mortalidad prenatal ${ }^{(1)}$, aunque se han descrito casos de sobrevivencia en varones con un genotipo XXY (Síndrome de Klinefelter) o mosaicismos somáticos (alteración genética en la que, en un mismo individuo, coexisten dos o más poblaciones de células con distinto genotipo) ${ }^{(8,9)}$.

La incidencia aproximada es de 1 por cada 50 000 nacidos vivos ${ }^{(10)}$. No se registran datos actuales sobre su prevalencia, pero se reporta que puede aparecer en todos los grupos poblacionales, con un predominio en personas de tez blanca ${ }^{(11,12)}$.

Las principales manifestaciones clínicas se evidencian a nivel cutáneo, por eso que es manejado a nivel dermatológico para su diagnóstico; sin embargo, también se presentan alteraciones a nivel ocular, pelo, uñas, sistema nervioso central, a nivel dentario, entre otros $(3,9,12)$.

A nivel bucal, los dientes se ven afectados entre un 50 a $80 \%$ en los pacientes; se presenta un retraso en la erupción dental, agenesia parcial o total de los dientes, morfología atípica dentaria de los tipos: conoidal, trapezoidal, clavija, tamaño pequeño y cúspides accesorias. También se suelen encontrar dientes impactados y maloclusiones ${ }^{(13)}$; en cuanto a éstas se ha encontrado una mayor tendencia a que los pacientes con este síndrome desarrollen una maloclusión de tipo clase III debido a una rotación de la man- díbula por la necesidad de cubrir la pérdida dentaria ${ }^{(14)}$.

Se considera importante la prevención de las enfermedades bucales en este tipo de pacientes, haciendo énfasis en su higiene oral, así mismo en la consistencia de la dieta, la frecuencia de ingesta de azúcares y en la exigencia de controles periódicos al dentista para el mantenimiento de la salud oral ${ }^{(3)}$. En el presente reporte se presenta un caso clínico del Síndrome de Bloch - Sulzberger, sin complicaciones, se discuten las manifestaciones orales, cuadro clínico y se revisa la literatura.

\section{Descripción del caso}

Paciente femenina de 13 años procedente de Talara (Piura, Perú). Fue remitida al servicio de Odontopediatría del Instituto de Salud Oral del Hospital Central de la Fuerza Aérea del Perú (FAP) en setiembre del 2018; con un historial de ausencia múltiple de piezas dentarias desde la infancia. La paciente refirió que nunca le erupcionaron las piezas dentarias de la zona posteroinferior izquierda y que además presentó alteraciones morfológicas en varios dientes.

En el año 2007, fue derivada por Neurología al servicio de Odontopediatría, durante la evaluación intraoral se evidenció 14 piezas dentarias, reborde alveolar derecho delgado, piezas anteriores giroversadas y una mordida anterior invertida.

\section{Características clínicas}

Mediante la historia clínica, se conoció que, debido al entrecruzamiento anterior, se le realizó un tratamiento ortopédico con una placa Hawley con tornillo de expansión y ganchos vestibulares para la retracción maxilar (Fig. 1A y 1B), como consecuencia se generó un descruce anterior y se ganó perímetro de arco para la erupción de las piezas permanentes. Además, en el año 2011, se le realizó una prótesis parcial removible provisional para devolver la funcionalidad y estética de la paciente. Actualmente, la paciente acude para el recambio de la prótesis. 
Su historial médico no refirió hábitos nocivos ni patológicos; sin embargo, en el año 2009 el servicio de Dermatología del Hospital Central FAP reportó lesiones hiperpigmentadas de forma lineal en brazos, extremidades y en la región nasal, no se evidenció alteraciones oculares ni ungueales. La valoración general evidenció que era una paciente hemodinámicamente estable, con las funciones vitales (presión arterial, pulso, frecuencia respiratoria y temperatura) dentro de los valores normales, y además se encontraba asintomática; en la evaluación dermatológica actual se observó lesiones cicatrizales en la región nasal (Fig. 2A, 2B y 2C).

Para el examen intraoral se le realizó un odontograma de rutina, se evidenció la ausencia de piezas dentarias (premolares y molares) en el sector posteroinferior, mientras que ausencia de premolares superiores en el lado izquierdo (Fig.
2D y 2E); alteraciones morfológicas dentarias y la persistencia de dientes primarios (Fig. 3A, 3B y 3C). Además, los rebordes se presentaron reducidos en altura y grosor; durante la palpación refirió ligero dolor localizado y de corta duración en la zona posteroinferior derecha. Al examen periodontal, se observó un buen estado de salud gingival.

\section{Características radiográficas}

Se realizó la toma de una radiografía panorámica y se visualizó asimetría de cuerpos mandibulares, ligera neumatización del seno maxilar, tabique nasal y cornetes aparentemente normales; a nivel dentario se encontró presencia de dientes temporales, agenesia de dientes permanentes, retraso en la maduración apical, retraso

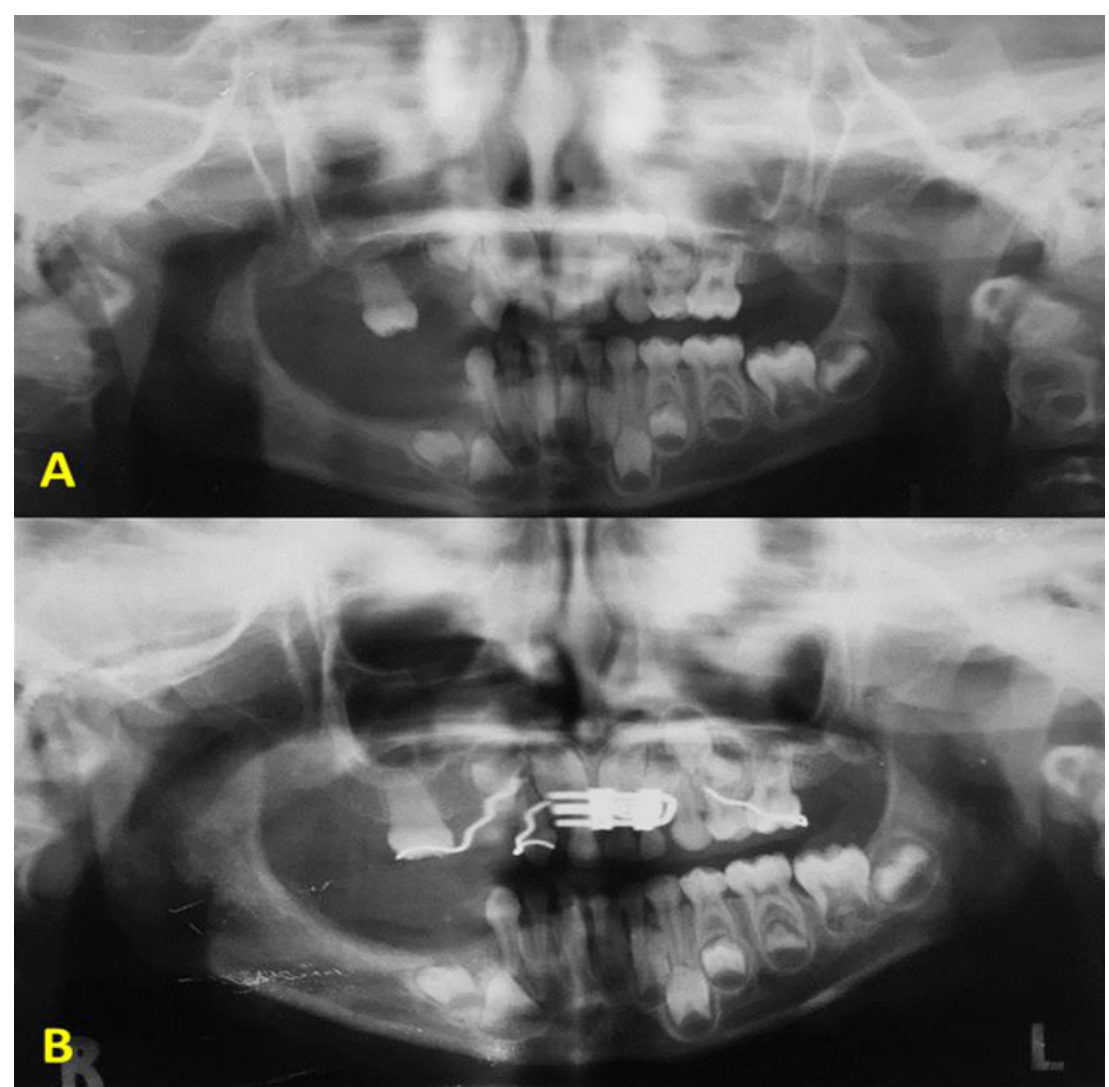

Fig. 1: A) Radiografía panorámica, año 2007 previo a la colocación del aparato ortopédico. B) Toma radiográfica del aparato ortopédico con tornillo de expansión, posterior a la fase de disyunción palatina. 


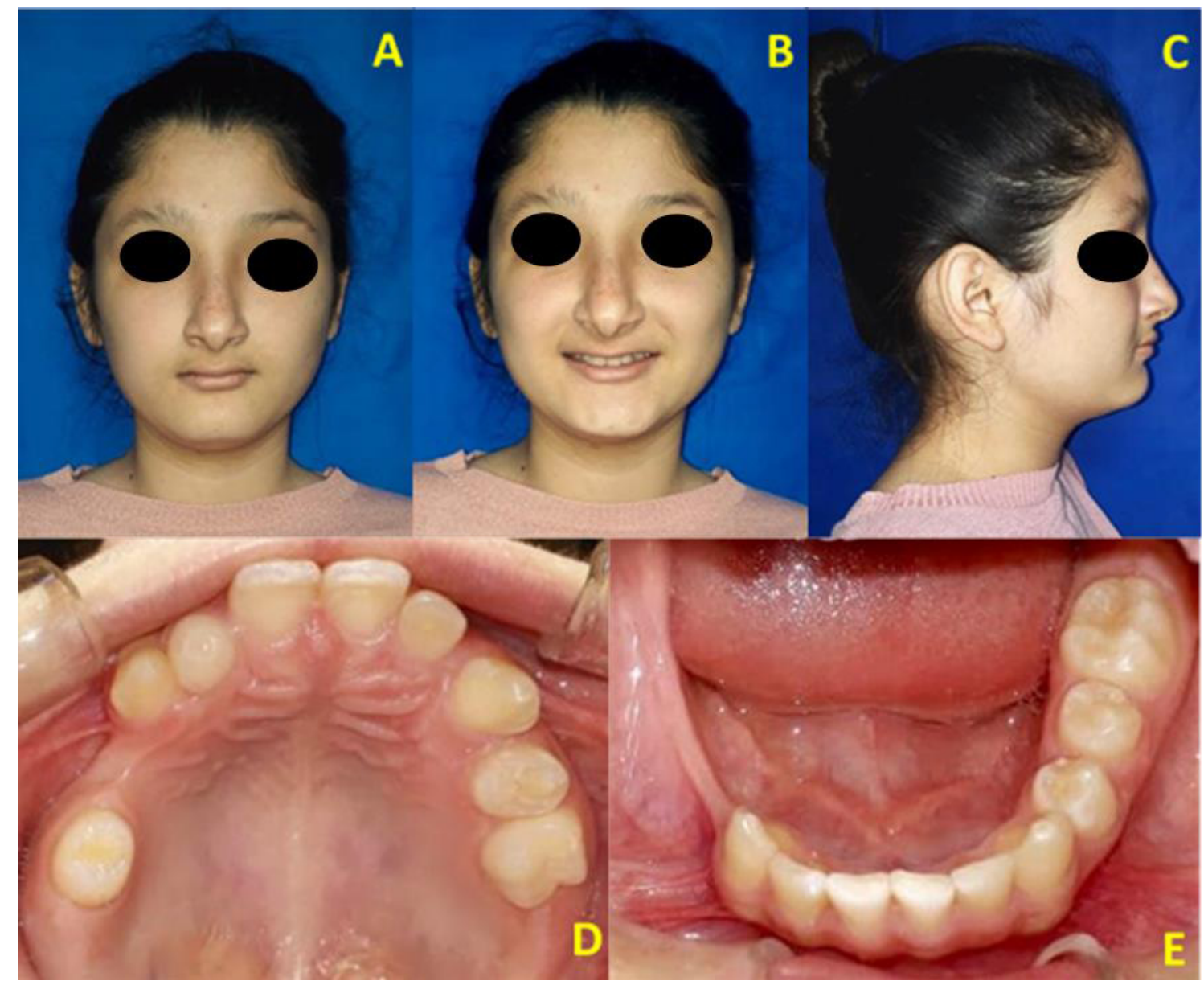

Fig. 2: A, B) Lesiones atróficas en la región nasal que se presentan de forma acumulada. C) Mentón protruído, región malar deprimida. D) Ausencia dentaria múltiple, alteraciones morfológicas y persistencia de dientes deciduos. E) Reborde alveolar delgado a nivel de la zona inferior derecha.

de cronología de erupción y una pieza dentaria retenida (Fig. 3D).

\section{Diagnóstico}

El diagnóstico médico definitivo otorgado desde la infancia fue el Síndrome de Bloch - Sulzberger (incontinencia pigmentaria), el cual fue compatible con las manifestaciones cutáneas y extracutáneas (dentales) observadas en la paciente.

Se procedió a planificar un tratamiento integral y multiespecializado para realizar la exodoncia de la pieza retenida en la zona posteroinferior izquierda, además de un manejo ortodóncico protésico para la rehabilitación de la función masticatoria y estética de la paciente.

\section{Discusión}

El presente reporte de caso evidenció las manifestaciones orales más comunes del Síndrome de Bloch - Sulzberger; una rara alteración hereditaria dominante ligada al cromosoma $\mathrm{X}^{(1)}$.

La etiología de este síndrome implica un mosaicismo pigmentario, el cual anteriormente era mencionado de manera equivocada en dos fases: la IP “esporádica” (o IP1) que se asociaba a 


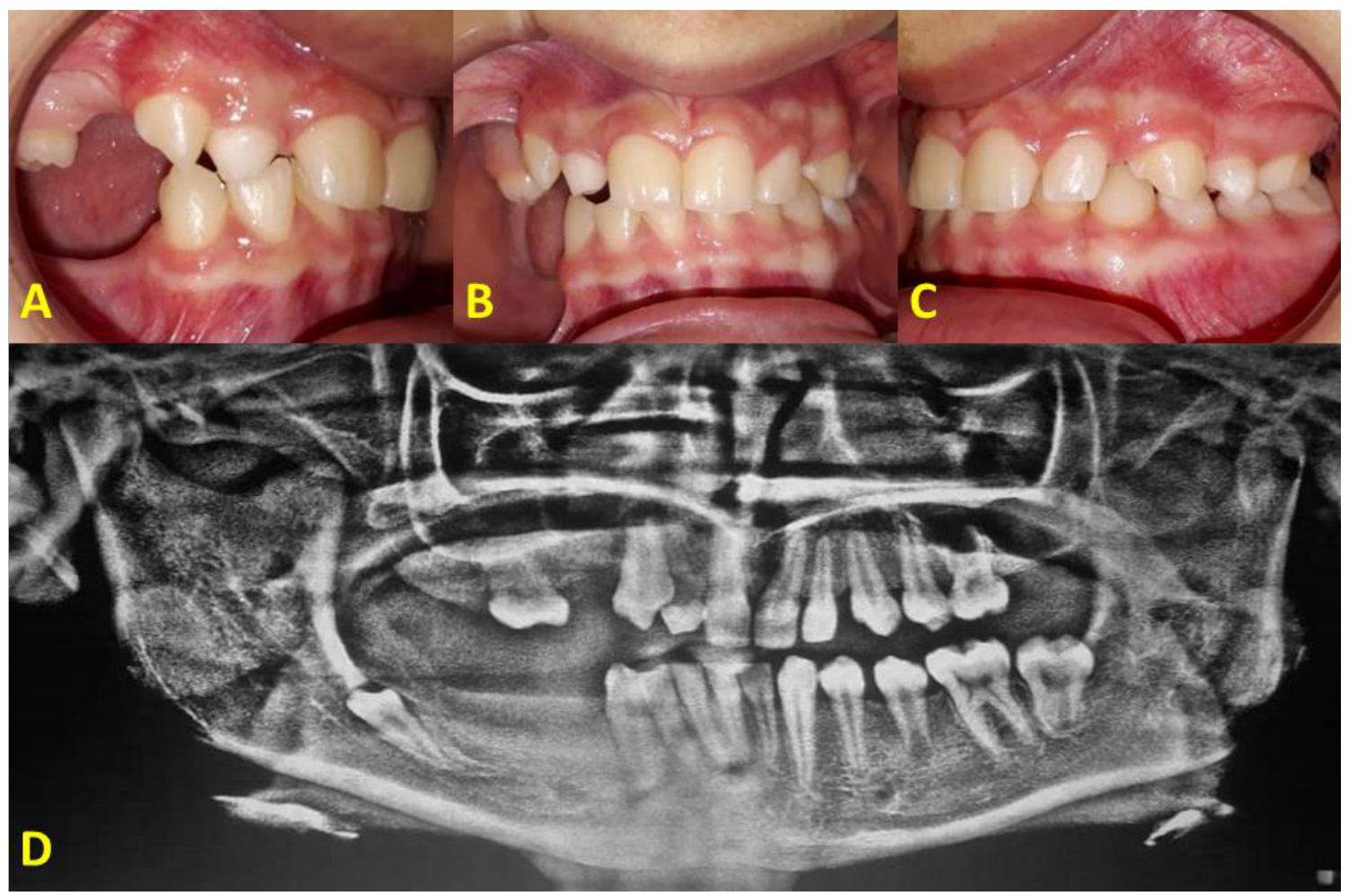

Fig. 3: A) Agenesia y rebordes disminuidos en los sextantes 1 y 6. B) Asimetría intraoral por la ausencia dentaria. C) Acomodación oclusal. D) Radiografía panorámica actual.

una mutación en el locus Xp11, y la "congénita" (o IP2), asociada a la región Xp28.20 ${ }^{(15)}$.

En el presente caso clínico se presentó las características diagnósticas bucales de la incontinencia pigmentaria. Se presentaron los signos típicos de hipodoncias y dientes cónicos; anomalías dentarias de número y forma que suelen ser un hallazgo sin mayor implicancias clínicas en la mayoría de casos.

Para diagnosticarlo, es fundamental el examen clínico (criterios mayores y menores); exámenes de sangre (se suele encontrar elevados niveles de $\operatorname{IgE}$, leucocitosis, eosinofilia, así como defectos en la quimiotaxis); estudios anatomopatológicos (para su confirmación y observación de los granos de melanina libres en la zona de hiperpigmentación, "espongliosis eosinofílica”, así como disqueratosis); también se recomienda un análisis genético molecular alternativo (estudio molecular del gen IKBKG (NEMO) locus Xq28) ${ }^{(3)}$.

Los criterios mayores (manifestaciones cutáneas), presentan una evolución que se divide en cuatro estadios: 1. Vesículoampolloso o inflamatorio, 2. Verrugoso, 3. Hiperpigmentado, 4. De cicatrizado o hipopigmentado o también denominado atrófico; cada uno presenta lesiones cutáneas características, aunque suelen coexistir y no se desarrollan secuencialmente en todos los pacientes. Las lesiones son más comunes a nivel de extremidades y el tronco; las zonas menos afectadas son los pliegues de flexión y la cara.

Los criterios menores (manifestaciones extracutáneas), se han reportado hasta en un $80 \%$ de casos, pero son menos comunes en los varones (probablemente debido al mosaicismo) ${ }^{(12)}$. Estos criterios implican: 
a) Pelo: Se presentan hasta en un 50\%. Pérdida de pelo a nivel de la coronilla de la cabeza (28 a 50\%). Se aprecia también agenesia de cejas y aplasia parcial de las glándulas sudoríparas ${ }^{(6)}$.

b) Uñas (manos y pies): Se suele afectar en un $40 \%$ de los casos de forma leve y transitoria; también se presentan arrugas, hoyos $y$ onicogrifosis.

c) Visión: El 35 a 77\% no presentan alteraciones oculares, las más comunes son en la retina (60\%), estrabismo (18-43\%), también disminución de agudeza visual y ceguera. Muchas veces miopía (46\%) o hipermetropía (37\%); la coriorretinitis exudativa, es la patología clásica.

d) Sistema nervioso central: Represetan del 30 al 50\%, se aprecia alteraciones psicomotrices, hidrocefalia, atrofia cerebral y retraso mental ${ }^{(12)}$.

e) Dientes: Son las manifestaciones más frecuentes, hasta en un $80 \%$ de los casos. Se afectan tanto la dentición temporal como la definitiva. Se caracteriza por: retraso en la erupción de dientes (18\%), agenesias parciales $(43 \%)$, anomalías de forma (30\%): conoidal, trapezoidal, en clavija, microdoncia, cúspides accesorias. Radiográficamente se observan dientes incluidos. La mayoría de pacientes presentan una maloclusión de clase III con disminución de la dimensión vertical y rotación mandibular. La constitución y estructura interna del diente y esmalte es normal $^{(3,6,12,16) \text {. }}$

El diagnóstico del presente caso se realizó a través de una evaluación interdisciplinaria que incluyó las consultas a los servicios de Dermatología, Neurología y Odontopediatría. Esto debido a que el síndrome al ser una enfermedad hereditaria requiere un historial familiar detallado y un examen físico completo si no fueron realizados en otros servicios; puede acompañarse el examen con un análisis genético.

El caso clínico presentado manifestó lesiones típicas de los diferentes estadios de la IP (lesiones hiperqueratósicas, máculas café- grisáceas acumuladas, vesículas y máculas hipopigementadas en diferentes partes del cuerpo). No se evidenciaron afecciones neurológicas y oculares.

Para el manejo odontológico, se recomienda un abordaje interdisciplinario entre las diferentes especialidades:

- Se debe priorizar la prevención y cuidado de la salud bucal mediante una rigurosa higiene oral, correcto cepillado dental y uso adecuado de hilo dental.

- Control de la dieta: en su consistencia física (relacionado a su adhesividad), en su alto índice de cariogeneidad (sobre todo por las noches al descender la secreción salival) y frecuencia de ingesta de alimentos cariogénicos.

Se debe evitar productos azucarados muy refinados, aquellos que faciliten el acúmulo de biofilm para la aparición y desarrollo de lesiones cariosas y enfermedad periodontal. Se debe insistir en visitas periódicas al dentista, obturaciones, aplicaciones tópicas de flúor y utilización de colutorios ${ }^{(3)}$.

Las alternativas terapéuticas dependerán de la etiología. En etapas tempranas el tratamiento ortopédico puede incluir el uso de mentoneras, máscaras faciales y/o reguladores de función clase III; en etapas más avanzadas se puede añadir, además del tratamiento ortopédico, alternativas quirúrgicas para corregir situaciones de dismorfías esqueléticas severas ${ }^{(3,12,17,18)}$.

La ausencia parcial de algunos dientes puede beneficiar el tratamiento ortodóntico al modificar la posición de otros dientes ya sea abriendo espacios o cerrando diastemas. También se pueden utilizar como pilares para prótesis parciales, utilizar implantes o minitornillos autorroscantes de titanio, como anclaje para movimientos retrusivos, intrusivos ${ }^{(19,20)}$.

Se indican también prótesis removibles con dientes de acrílico para la reconstitución estética hasta lograr un mayor desarrollo del hueso maxilar hasta que se pueda realizar una prótesis implantosoportada. En adultos se puede 
indicar prótesis convencionales fijas, adhesivas e incluso implantes dentales; en estos casos es imprescindible identificar patrones familiares que permita detectar la mutación ${ }^{(3)}$.

En el presente caso se adaptó la prótesis parcial removible que ya tenía la paciente; esto para permitir la restitución funcional de las arcadas dentarias con el fin de mantener el espacio y dimensión vertical de la oclusión, facilitar la masticación, evitar interferir con el propio desarrollo del niño y permitir un crecimiento armónico.

\section{Conclusiones}

El Síndrome de Bloch - Sulzberger (incontinencia pigmentaria) es una enfermedad genética poco común. Sus principales signos clínicos se manifiestan en la piel cuyas manifestaciones más sobresalientes se encuentran en la piel; sin embargo, las principales manifestaciones extracutáneas se encuentran en la cavidad bucal y ambas son la base del diagnóstico. Los signos neurológicos constituyen los más graves de la enfermedad y son el principal factor de pronóstico. En el presente caso se utilizó la combinación del examen clínico, la revisión de antecedentes (historia clínica) y el uso de la radiografía para permitir un tratamiento funcional y estético que impacte de forma positiva su calidad de vida y relaciones psicosociales.

\section{Contribución de autoría}

1. Concepción y diseño del estudio

2. Adquisición de datos

3. Análisis de datos

4. Discusión de los resultados

5. Redacción del manuscrito

6. Aprobación de la versión final del manuscrito

YCR ha contribuido en: 1, 3, 5 y 6 .

JCQ ha contribuido en: 2, 4 y 6 .

EJG ha contribuido en: 2, 4, 5 y 6 .

\section{Referencias}

1. Mühlenstädt E, Eigelshoven S, Hoff NP, Reifenberger J, Homey B, Bruch-Gerharz D. Bloch-Sulzberger syndrome. Hautarzt. 2010; 61 (10): 831-3.

2. Morrell DS, Burkhart CN, Siegel D. Enfermedades hereditarias seleccionadas. En: Eichenfield L, Frieden I, Esterly N. (eds.). Dermatologia Neonatal. 2da ed. Barcelona, España: Elsevier; 2009. p. 485.

3. Pipa VA, González GM, López-Arranz ME, Fernández TJ. Incontinencia Pigmentaria. Consideraciones odontoestomatológicas: Profilaxis y terapéutica. Av Odontoestomatol. 2005; 21 (4): 211-5.

4. García DJ, De Unamuno P, Fernández LE, Salazar VJ, Armijo M. Incontinentia pigmenti: XXY male with family history. Clin Genet. 1990; 38 (2): 128-38.

5. Ormerod AD, White MI, McKay E, Johnston AW. Incontinentia pigmenti in a boy with Klinefelter's syndrome. J Med Genet. 1987; 24 (7): 39-41.

6. Landy SJ, Donnai D. Incontinentia pigmenti (Bloch-Sulzberger syndrome). J Med Genet. 1993; 30 (1): 53-9.

7. Geiges ML. Traces of Marion B. Sulzberger in the Museum of Wax Moulages in Zurich and their importance for the history of dermatology. J Am Acad Dermatol.2009; 60 (6): 980-4.

8. The International Incontinentia Pigmenti (IP) Consortium. Genomic rearrangement in NEMO impairs NF-(kappa)B activation and is a cause of incontinentia pigmenti. Nature. 2000;405 (6785): 466-72.

9. Kenwrick S, Woffendin H, Jakins T, Shuttleworth SG, Mayer E, Greenhalgh L, Whittaker J, Rugolotto S, Bardaro T, Esposito T, D'Urso M, Soli F, Turco A, Smahi A, Hamel-Teillac D, Lyonnet S, Bonnefont JP, Munnich A, Aradhya S, Kashork CD, Shaffer LG, Nelson DL, Levy M, Lewis RA; International IP Consortium. Survival of male patients with incontinentia pigmenti carrying a lethal mutation can be explained by somatic mosaicism or Klinefelter syndrome. Am J Hum Genet. 2001; 69 (6): 1210-7.

10. Chang JT, Chiu PC, Chen YY, Wang HP, Hsieh KS. Multiple clinical manifestations and 
diagnostic challenges of incontinentia pigmenti - 12 years' experience in 1 medical center. J Chin Med Assoc. 2008; 71(9): 455-60.

11. Carney RG. Incontinentia pigmenti: A world statistical analysis. Arch Dermatol.1976; 112 (4): 535-42.

12. Gómez VR, Juárez M, Plascencia GA, Reyes MG, Saénz CC, Káram M et al. Incontinentia pigmenti. Artículo de revisión. Dermat Cosmet Méd Quir. 2010;8 (2): 104-9.

13. Rouse JS. Incontinentia pigmenti: overcoming cosmetic challenges. Compend Contin Educ Dent.1999; 20 (11): 1034-6.

14. Rouse JS. Incontinentia pigmenti: overcoming cosmetic challenges. Compend Contin Educ Dent. 1999; 20 (11): 1040-1.

15. Berlin AL, Paler AS, Chan LS. Incontinentia pigmenti: A review and update on the molecular basis of pathophysiology. J Am Acad Dermatol. 2002; 47(2): 169-87.
16. Kasmann KB, Jurin BB, Ruprech KW. Incontinentia pigmenti (Bloch-Schulzberger syndrome): case report and differential diagnosis to related dermato-ocular syndroms. Ophthalmologica. 1999; 213 (1): 63-9.

17. Romero A, Tufińo M, Villacís A, Salazar M. Incontinencia pigmentaria o síndrome de Bloch-Sulzberger. Dermatol Rev Mex. 2014; 58 (6): 539-43.

18. Atamari AN, Solórzano GS. Incontinentia pigmenti (Síndrome de BlochSulzberger) en un paciente varón. Reporte de un caso. Rev Med Hered. 2015;26 (4): 238-41.

19. Maino BG, Pagin P, Mura P. Spider Screw ${ }^{\circledR}$ Anclaje absoluto de carga inmediata. Rev Esp Ortod. 2003;33 (1): 21-30.

20. Puigdollers A. Anclaje estable sobre implantes palatinos. Rev Esp Ortod. 2003; 33 (2): 121-31.

21. De Rábago JA, Sanz M. Manejo multidisciplinario de las agenesias dentarias. Periodoncia. 2003;13 (5): 399-412.

Yuri Castro Rodríguez: yuricastro_16@hotmail.com 